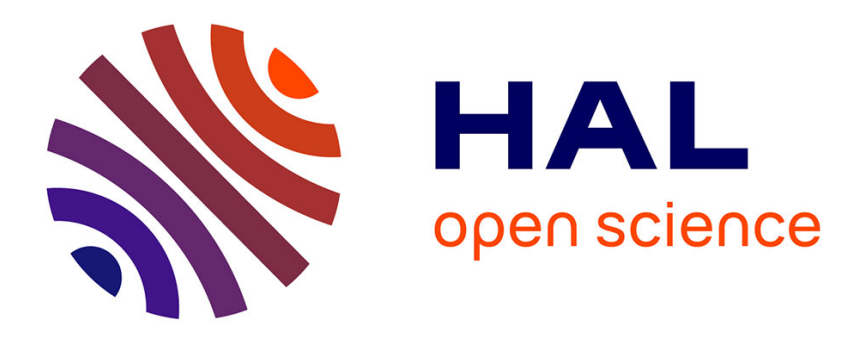

\title{
An Application of Martin-Löf Randomness to Effective Probability Theory
}

Mathieu Hoyrup, Cristobal Rojas

\section{To cite this version:}

Mathieu Hoyrup, Cristobal Rojas. An Application of Martin-Löf Randomness to Effective Probability Theory. 5th Conference on Computability in Europe - CiE 2009, Jul 2009, Heidelberg, Germany. pp.260-269, 10.1007/978-3-642-03073-4 . hal-00425556

\section{HAL Id: hal-00425556 https://hal.science/hal-00425556}

Submitted on 22 Oct 2009

HAL is a multi-disciplinary open access archive for the deposit and dissemination of scientific research documents, whether they are published or not. The documents may come from teaching and research institutions in France or abroad, or from public or private research centers.
L'archive ouverte pluridisciplinaire HAL, est destinée au dépôt et à la diffusion de documents scientifiques de niveau recherche, publiés ou non, émanant des établissements d'enseignement et de recherche français ou étrangers, des laboratoires publics ou privés. 


\title{
An Application of Martin-Löf Randomness to Effective Probability Theory
}

\author{
Mathieu Hoyrup ${ }^{1}$ and Cristóbal Rojas ${ }^{2}$ \\ 1 LORIA - 615, rue du jardin botanique, BP 239 \\ 54506 Vandœuvre-lès-Nancy, FRANCE, hoyrup@loria.fr \\ 2 Institut de Mathématiques de Luminy, Campus de Luminy, Case 907 \\ 13288 Marseille Cedex 9, FRANCE, rojas@iml.univ-mrs.fr
}

\begin{abstract}
In this paper we provide a framework for computable analysis of measure, probability and integration theories. We work on computable metric spaces with computable Borel probability measures. We introduce and study the framework of layerwise computability which lies on Martin-Löf randomness and the existence of a universal randomness test. We then prove characterizations of effective notions of measurability and integrability in terms of layerwise computability. On the one hand it gives a simple way of handling effective measure theory, on the other hand it provides powerful tools to study Martin-Löf randomness, as illustrated in a sequel paper.

Keywords. Algorithmic randomness, universal test, computable analysis, effective probability theory, Lebesgue integration, layerwise computability.
\end{abstract}

\section{Introduction}

While computability on topological spaces is now well-established (see [1,2] e.g.), the landscape for computability on measurable spaces and probability spaces is rather uneven. An effective presentation of measurable spaces is proposed in [3]. Computability on $L^{p}$-spaces has been studied in [4-6] for euclidean spaces with the Lebesgue measure. Computability of measurable sets has been studied, on the real line with the Lebesgue measure in [7] and on second countable locally compact Hausdorff spaces with a computable $\sigma$-finite measure in [8]. In the latter article a computability framework for bounded integrable functions is also introduced, when the measure is finite. Another approach based on probabilistic computing has been recently developed in [9]. The connection of this with the previous mentioned works remains to be established.

On the other hand, another effective approach to probability theory has already been deeply investigated, namely algorithmic randomness, as introduced by Martin-Löf in [10]. This theory was originally developed on the Cantor space, i.e. the space of infinite binary sequences, endowed with a computable probability measure. Since then, the theory has been mainly studied on the Cantor space from the point of view of recursion theory, focused on the interaction between 
randomness and reducibility degrees. The theory has been recently extended to more general spaces in [11-13].

In this paper, we propose a general unified framework for the computable analysis of measure and integration theory, and establish intimate relations with algorithmic randomness. We first consider two natural ways (more or less already present in the literature) of giving effective versions of the notions of measurable set, measurable map and integrable function.

Then we develop a third approach which we call layerwise computability that is based on the existence of a universal randomness test. This fundamental result proved by Martin-Löf in his seminal paper is a peculiarity of the effective approach of mathematics, having no counterpart in the classical world. Making a systematic use of this has quite unexpected strong consequences: (i) it gives topological characterizations of effective measurability notions; (ii) measure-theoretic notions, usually defined almost everywhere, become set-theoretic when restricting to effective objects; (iii) the practice of these notions is rather light: most of the basic manipulations on computability notions on topological spaces can be straightforwardly transposed to effective measurability notions, by the simple insertion of the term "layerwise". This language trick may look suspicious, but in a sense this paper provides the background for this to make sense and being practiced.

In this way, Martin-Löf randomness and the existence of a universal test find an application in computable analysis. In [14] we show how this framework in turn provides powerful tools to the study of algorithmic randomness, extending Birkhoff's ergodic theorem for random points from computable functions to effectively measurable ones in a simple way thanks to layerwise computability. In [14] we also show that this framework provides a general way of deriving results in the spirit of [15].

In Sect. 2 we recall the background on computable probability spaces and define the notion of layering of the space, which will be the cornerstone of our approach. In Sect. 3 we present two approaches to make measure-theoretical notions on computable probability space effective. Some definitions are direct adaptations of preceding works, some others are new (in particular the notions of effectively measurable maps and effectively integrable functions). In Sect. 4 we present our main contribution, namely layerwise computability, and state several characterizations. Being rather long, the proofs are gathered in the appendix.

\section{Preliminaries}

Computable metric space. Let us first recall some basic results established in $[12,13]$. We work on the well-studied computable metric spaces (see $[1,2,16,17])$.

Definition 1. A computable metric space is a triple $(X, d, \mathcal{S})$ where:

1. $(X, d)$ is a separable metric space,

2. $\mathcal{S}=\left\{s_{i}: i \in \mathbb{N}\right\}$ is a countable dense subset of $X$ with a fixed numbering,

3. $d\left(s_{i}, s_{j}\right)$ are uniformly computable real numbers. 
$\mathcal{S}$ is called the set of $\boldsymbol{i d e a l}$ points. If $x \in X$ and $r>0$, the metric ball $B(x, r)$ is defined as $\{y \in X: d(x, y)<r\}$. The set $\mathcal{B}:=\{B(s, q): s \in \mathcal{S}, q \in \mathbb{Q}, q>0\}$ of ideal balls, which is a basis of the topology, has a canonical numbering $\mathcal{B}=\left\{B_{i}: i \in \mathbb{N}\right\}$. An effective open set is an open set $U$ such that there is a r.e. set $E \subseteq \mathbb{N}$ with $U=\bigcup_{i \in E} B_{i}$. If $B_{i}=B(s, r)$ we denote by $\bar{B}_{i}$ the closed ball $\bar{B}(s, r)=\{x \in X: d(x, s) \leq r\}$. The complement of $\bar{B}_{i}$ is effectively open, uniformly in $i$. If $X^{\prime}$ is another computable metric space, a function $f: X \rightarrow$ $X^{\prime}$ is computable if the sets $f^{-1}\left(B_{i}^{\prime}\right)$ are effectively open, uniformly in $i$. Let $\overline{\mathbb{R}}:=\mathbb{R} \cup\{-\infty,+\infty\}$. A function $f: X \rightarrow \overline{\mathbb{R}}$ is lower (resp. upper) semicomputable if $f^{-1}\left(q_{i},+\infty\right]$ (resp. $f^{-1}\left[-\infty, q_{i}\right)$ ) is effectively open, uniformly in $i$ (where $q_{0}, q_{1}, \ldots$ is a fixed effective enumeration of the set of rational numbers Q). We remind the reader that there is an effective enumeration $\left(f_{i}\right)_{i \in \mathbb{N}}$ of all the lower semi-computable functions $f: X \rightarrow[0,+\infty]$.

A numbered basis $\mathcal{B}^{\prime}=\left\{B_{0}^{\prime}, B_{1}^{\prime}, \ldots\right\}$ of the topology is called effectively equivalent to $\mathcal{B}$ if every $B_{i}^{\prime} \in \mathcal{B}^{\prime}$ is effectively open uniformly in $i$, and every $B_{i} \in \mathcal{B}$ is an effective union of elements of $\mathcal{B}^{\prime}$, uniformly in $i$.

Computable probability space. In [3] is studied an effective version of measurable spaces. Here, we restrict our attention to metric spaces endowed with the Borel $\sigma$-field (the $\sigma$-field generated by the open sets).

Let $(X, d, \mathcal{S})$ be a computable metric space. We first recall what it means for a Borel probability measure over $X$ to be computable. Several equivalent approaches can be found in $[12,13,18,19]$ for instance.

Definition 2. A Borel probability measure $\mu$ is computable if $\mu\left(B_{i_{1}} \cup \ldots \cup B_{i_{n}}\right)$ are lower semi-computable, uniformly in $i_{1}, \ldots, i_{n}$.

In $[18,19]$ it is proved that $\mu$ is computable if and only if $\int f_{i} \mathrm{~d} \mu$ are lower semicomputable, uniformly in $i$ (where $f_{i}$ are the lower semi-computable functions).

Proposition 1. Let $\mu$ be a computable Borel probability measure. If $f: X \rightarrow$ $[0,+\infty)$ is upper semi-computable and bounded by $M$ then $\int f \mathrm{~d} \mu$ is upper semicomputable (uniformly in a description of $f$ and $M$ ).

Following [13] we introduce:

Definition 3 (from [13]). A computable probability space is a pair $(X, \mu)$ where $X$ is a computable metric space and $\mu$ is a computable Borel probability measure on $X$.

From now and beyond, we will always work on computable probability spaces.

A ball $B(s, r)$ is said to be a $\boldsymbol{\mu}$-almost decidable ball if $r$ is a computable positive real number and $\mu(\{x: d(s, x)=r\})=0$. The following result has been independently proved in [9] and [13].

Theorem 1. Let $(X, \mu)$ be a computable probability space. There is a basis $\mathcal{B}^{\mu}=$ $\left\{B_{1}^{\mu}, B_{2}^{\mu}, \ldots\right\}$ of uniformly $\mu$-almost decidable balls which is effectively equivalent to the basis $\mathcal{B}$ of ideal balls. The measures of their finite unions are then uniformly computable. 
Algorithmic randomness. Here, $(X, \mu)$ is a computable probability space. Martin-Löf randomness was first defined in [10] on the space of infinite symbolic sequences. Generalizations to abstract spaces have been investigated in [11-13, 20]. We follow the latter two approaches, developed on computable metric spaces.

Definition 4. A Martin-Löf test (ML-test) is a sequence of uniformly effective open sets $U_{n}$ such that $\mu\left(U_{n}\right)<2^{-n}$.

A point $x$ passes $a M L$-test $U$ if $x \notin \bigcap_{n} U_{n}$. A point is Martin-Löf random (ML-random) if it passes all ML-tests. We denote the set of $M L$-random points by $M L_{\mu}$.

If a set $A \subseteq X$ can be enclosed in a ML-test $\left(U_{n}\right)$, i.e. $A \subseteq \bigcap_{n} U_{n}$ then we say that $A$ is an effective $\boldsymbol{\mu}$-null set.

The following fundamental result, proved by Martin-Löf on the Cantor space with a computable probability measure, can be extended to any computable probability space using Thm. 1 (almost decidable balls behave in some way as the cylinders in the Cantor space, as their measures are computable).

Theorem 2 (adapted from [10]). Every computable probability space $(X, \mu)$ admits a universal Martin-Löf test, i.e. a $M L$-test $U$ such that for all $x \in X, x$ is $M L$-random $\Longleftrightarrow x$ passes the test $U$. Moreover, for each $M L$-test $V$ there is a constant c (computable from a description of $V$ ) such that $V_{n+c} \subseteq U_{n}$ for all $n$.

Definition 5. Let $(X, \mu)$ be a computable probability space. Let $\left(U_{n}\right)_{n \in \mathbb{N}}$ be a universal $M L$-test. We call $K_{n}:=X \backslash U_{n}$ the $\boldsymbol{n}^{\text {th }}$ layer of the space and the sequence $\left(K_{n}\right)_{n \in \mathbb{N}}$ the layering of the space.

One can suppose w.l.o.g. that the universal test is decreasing: $U_{n+1} \subseteq U_{n}$. Hence the set $\mathrm{ML}_{\mu}$ of ML-random points can be expressed as an increasing union: $\mathrm{ML}_{\mu}=\bigcup_{n} K_{n}$. In [14] we prove that the sets $K_{n}$ are compact, in an effective way, which justifies their name.

\section{Effective versions of measurability notions}

We now consider effective versions of the notions of measurable set, measurable map, and integrable function.

\subsection{The approach up to null sets}

This approach is by equivalence classes. As a concequence, the obtained definitions cannot distinguish between objects which coincide up to a null set. 
Measurable sets. This approach to computability of measurable sets was first proposed by STanin [7] on $\mathbb{R}$ with the Lebesgue measure, and generalized by Edalat [8] to any second countable locally compact Hausdorff spaces with a computable regular $\sigma$-finite measure. We present the adaptation of this approach to computable probability spaces (which are not necessarily locally compact).

Let $(X, \mu)$ be a computable probability space and $\mathfrak{S}$ the set of Borel subsets of $X$. The function $d_{\mu}: \mathfrak{S}^{2} \rightarrow[0,1]$ defined by $d_{\mu}(A, B)=\mu(A \Delta B)$ for all Borel sets $A, B$ is a pseudo-metric. Let $[\mathfrak{S}]_{\mu}$ be the quotient of $\mathfrak{S}$ by the equivalence relation $A \sim_{\mu} B \Longleftrightarrow d_{\mu}(A, B)=0$ and $\mathcal{A}_{\mu}$ be the set of finite unions of $\mu$-almost decidable balls from $\mathcal{B}^{\mu}$ with a natural numbering $\mathcal{A}_{\mu}=\left\{A_{1}, A_{2}, \ldots\right\}$. We denote by $[A]_{\mu}$ the equivalence class of a Borel set $A$. The following result was proved in [21] (Thm. 2.3.2.1) for computable metric spaces.

Proposition 2. ([医 $\left.]_{\mu}, d_{\mu}, \mathcal{A}_{\mu}\right)$ is a computable metric space.

The following definition is then the straightforward adaptation of $[7,8]$.

Definition 6. A Borel set $A$ is called a $\boldsymbol{\mu}$-recursive set if its equivalence class $[A]_{\mu}$ is a computable point of the computable metric space $[\mathfrak{S}]_{\mu}$.

In other words, there is a total recursive function $\varphi: \mathbb{N} \rightarrow \mathbb{N}$ such that $\mu\left(A \Delta A_{\varphi(n)}\right)<2^{-n}$ for all $n$. The measure of any $\mu$-recursive set is computable. Observe that an ideal ball need not be $\mu$-recursive as its measure is in general only lower semi-computable. On the other hand, $\mu$-almost decidable balls are always $\mu$-recursive.

Measurable maps. Here $Y$ is a computable metric space. To the notion of $\mu$ recursive set corresponds a natural effective version of the notion of $\mu$-recursive map:

Definition 7. A measurable map $T:(X, \mu) \rightarrow Y$ is called a $\boldsymbol{\mu}$-recursive map if there exists a basis of balls $\hat{\mathcal{B}}=\left\{\hat{B}_{1}, \hat{B}_{2}, \ldots\right\}$ of $Y$, which is effectively equivalent to the basis of ideal balls $\mathcal{B}$, and such that $T^{-1}\left(\hat{B}_{i}\right)$ are uniformly $\mu$-recursive sets.

Integrable functions. Computability on $L^{p}$ spaces has been studied in [4-6] for euclidean spaces with the Lebesgue measure. The $L^{1}$ case can be easily generalized to any computable probability space, and a further generalization including $\sigma$-finite measures might be carried out without difficulties.

Let $(X, \mu)$ be a computable probability space. Let $\mathcal{F}$ be the set of measurable functions $f: X \rightarrow \overline{\mathbb{R}}$ which are integrable. Let $I_{\mu}: \mathcal{F} \times \mathcal{F} \rightarrow[0,+\infty)$ be defined by $I_{\mu}(f, g)=\int|f-g| \mathrm{d} \mu . I_{\mu}$ is a metric on the quotient space $L^{1}(X, \mu)$ with the relation $f \sim_{\mu} g \Longleftrightarrow I_{\mu}(f, g)=0$. There is a set $\mathcal{E}=\left\{f_{0}, f_{1}, \ldots\right\}$ of uniformly computable effectively bounded functions $\left(\left|f_{i}\right|<M_{i}\right.$ with $M_{i}$ computable from $i$ ) which is dense in $L^{1}(X, \mu)$ (this is a direct consequence of Prop. 2.1 in [12]). $\mathcal{E}$ is called the set of ideal functions. 
Proposition 3. $\left(L^{1}(X, \mu), d_{\mu}, \mathcal{E}\right)$ is a computable metric space.

This leads to a first effective notion of integrable function:

Definition 8. A function $f: X \rightarrow \overline{\mathbb{R}}$ is $\boldsymbol{\mu}$-recursively integrable if its equivalence class is a computable point of the space $L^{1}(X, \mu)$, i.e. $f$ can be effectively approximated by ideal functions in the $L^{1}$ norm.

If $f: X \rightarrow \overline{\mathbb{R}}$ is integrable, then $f$ is $\mu$-recursively integrable if and only if so are $f^{+}=\max (f, 0)$ and $f^{-}=\max (-f, 0)$.

\subsection{The approach up to effective null sets}

On a metric space, every Borel probability measure is regular, i.e. for every Borel set $A$ and every $\varepsilon>0$ there is a closed set $F$ and an open set $U$ such that $F \subseteq A \subseteq U$ and $\mu(U \backslash F)<\varepsilon$ (see [22]). It gives an alternative way to define an effective version of the notion of measurable set. We will see how to define effectively $\mu$-measurable maps and effectively $\mu$-integrable functions using the same idea.

Measurable sets. Edalat [8] already used regularity of measures to define $\mu$-computable sets, a notion that is stronger than $\mu$-recursivity. Let us consider the adaptation of this notion to computable probability spaces (for coherence reasons, we use the expression "effective $\mu$-measurability" instead of " $\mu$-computability").

Definition 9. $A$ Borel set $A$ is effectively $\boldsymbol{\mu}$-measurable if there are uniformly effective open sets $U_{i}, V_{i}$ such that $X \backslash V_{i} \subseteq A \subseteq U_{i}$ and $\mu\left(U_{i} \cap V_{i}\right)<2^{-i}$.

This is a generalization of the notion of effective $\mu$-null set (see after Def. 4): a set of measure zero is an effective $\mu$-null set if and only if it is effectively $\mu$-measurable.

Example 1. The whole space $X$ is effectively $\mu$-measurable. More generally, an effective open set is effectively $\mu$-measurable if and only if its measure is computable. The Smith-Volterra-Cantor set or fat Cantor set, which is an effective compact subset of $[0,1]$ whose Lebesgue measure is $1 / 2$, is effectively $\lambda$ measurable where $\lambda$ denotes the Lebesgue measure (see SVC(4) in [23]).

Measurable maps. To the notion of effectively $\mu$-measurable set corresponds a natural effective version of the notion of measurable map:

Definition 10. A measurable map $T:(X, \mu) \rightarrow Y$ is effectively $\boldsymbol{\mu}$-measurable if there exists a basis of balls $\hat{\mathcal{B}}=\left\{\hat{B}_{1}, \hat{B}_{2}, \ldots\right\}$ of $Y$, which is effectively equivalent to the basis of ideal balls $\mathcal{B}$, and such that $T^{-1}\left(\hat{B}_{i}\right)$ are uniformly effectively $\mu$-measurable sets. 
Integrable functions. In [8] a notion of $\mu$-computable integrable function is proposed: such a function can be effectively approximated from above and below by simple functions. This notion is developed on any second countable locally compact Hausdorff spaces endowed with a computable finite Borel measure. In this approach only bounded functions can be handled, as they are dominated by simple functions, which are bounded by definition. We overcome this problem, providing at the same time a framework for metric spaces that are not locally compact, as function spaces.

The following definition is a natural extension of the counterpart of Def. 9 for the characteristic function $\mathbf{1}_{A}$ of an effectively $\mu$-measurable set $A$.

Definition 11. A function $f: X \rightarrow[0,+\infty]$ is effectively $\boldsymbol{\mu}$-integrable if there are uniformly lower semi-computable functions $g_{n}: X \rightarrow[0,+\infty]$ and upper semi-computable functions $h_{n}: X \rightarrow[0,+\infty)$ such that:

1. $h_{n} \leq f \leq g_{n}$,

2. $\int\left(g_{n}-h_{n}\right) \mathrm{d} \mu<2^{-n}$,

3. $h_{n}$ is bounded by some $M_{n}$ which is computable from $n$.

Observe that a set $A$ is effectively $\mu$-measurable if and only if its characteristic function $\mathbf{1}_{A}$ is effectively $\mu$-integrable.

\section{The algorithmic randomness approach: layerwise computability}

\subsection{Layerwise computability}

We remind the reader that every computable probability space comes with a Martin-Löf layering $\left(K_{n}\right)_{n \in \mathbb{N}}$ (see Def. 5). In the following definition, $\mathcal{B}=\left\{B_{i}\right.$ : $i \in \mathbb{N}\}$ is the basis of ideal balls of $Y$.

Definition 12. A set $A$ is layerwise semi-decidable if it is semi-decidable on every $K_{n}$, uniformly in $n$. In other words, $A$ is layerwise semi-decidable if there are uniformly effective open sets $U_{n}$ such that $A \cap K_{n}=U_{n} \cap K_{n}$ for all $n$. $A$ set $A$ is layerwise decidable if both $A$ and its complement are layerwise semidecidable. A function $T:(X, \mu) \rightarrow Y$ is layerwise computable if $T^{-1}\left(B_{i}\right)$ are layerwise semi-decidable, uniformly in $i$.

In the language of representations, a set $A$ is layerwise semi-decidable (resp. layerwise decidable) if there is a machine which takes $n$ and a Cauchy representation of $x \in K_{n}$ as inputs, and eventually halts if and only if $x \in A$ (resp. halts and outputs 1 if $x \in A, 0$ if $x \notin A$ ) (if $x \notin K_{n}$, nothing is assumed about the behavior of the machine).

Actually, every computability notion on computable metric spaces has in principle its layerwise version. For instance one can define layerwise lower semicomputable functions $f: X \rightarrow \overline{\mathbb{R}}$.

Let us state some basic properties of layerwise computable maps, when considering the push-forward measure $\nu$ defined by $\nu(A)=\mu\left(T^{-1}(A)\right)$. 
Proposition 4. Let $T:(X, \mu) \rightarrow Y$ be a layerwise computable map.

- The push-forward measure $\nu:=\mu \circ T^{-1} \in \mathcal{M}(Y)$ is computable.

- T preserves $M L$-randomness, i.e. $T\left(M L_{\mu}\right) \subseteq M L_{\nu}$. Moreover, there is a constant c (computable from a description of $T$ ) such that $T\left(K_{n}\right) \subseteq K_{n+c}^{\prime}$ for all $n$, where $\left(K_{n}^{\prime}\right)$ is the canonical layering of $(Y, \nu)$.

- If $f:(Y, \nu) \rightarrow Z$ is layerwise computable then so is $f \circ T$.

- If $A \subseteq Y$ is layerwise decidable (resp. semi-decidable) then so is $T^{-1}(A)$.

The first point implies that in the particular case when $Y=\mathbb{R}$, a layerwise computable function is then a computable random variable as defined in [24]: its distribution $\nu$ over $\mathbb{R}$ is computable. Observe that when $\nu$ is the push-forward of $\mu$, layerwise computability notions interact as the corresponding plain computability ones; however, without this assumption on $\nu$ the last three points may not hold.

As shown by the following proposition, if layerwise computable objects differ at one ML-random point then they essentially differ, i.e. on a set of positive measure.

Proposition 5. Let $A, B \subseteq X$ be layerwise decidable sets and $T_{1}, T_{2}:(X, \mu) \rightarrow$ $Y$ layerwise computable functions.

- $A=B \bmod 0$ if and only if $A \cap M L_{\mu}=B \cap M L_{\mu}$.

- $T_{1}=T_{2}$ almost everywhere if and only if $T_{1}=T_{2}$ on $M L_{\mu}$.

\subsection{Characterizations of effective measure-theoretic notions in terms of layerwise computability}

Measurable sets. The notion of effective $\mu$-measurable set is strongly related to the Martin-Löf approach to randomness. Indeed, if $A$ is a Borel set such that $\mu(A)=0$ then $A$ is effectively $\mu$-measurable if and only if it is an effective $\mu$-null set. If $A$ is effectively $\mu$-measurable, coming with $C_{n}, U_{n}$, then $\bigcup_{n} C_{n}$ and $\bigcap_{n} U_{n}$ are two particular representatives of $[A]_{\mu}$ which coincide with $A$ on $\mathrm{ML}_{\mu}$. We can even go further, as the following result proves.

Theorem 3. Let $A$ be a Borel set. We have:

1. A is $\mu$-recursive $\Longleftrightarrow A$ is equivalent to an effectively $\mu$-measurable set.

2. $A$ is effectively $\mu$-measurable $\Longleftrightarrow A$ is layerwise decidable.

The equivalences are uniform. These characterizations enable one to use layerwise computability to simplify proofs: for instance basic operations that preserve decidability of sets, as finite unions or complementation, also preserve layerwise computability in a straightforward way, hence they preserve $\mu$-recursivity and effective $\mu$-measurability.

Let $A$ be a $\mu$-recursive set: it is equivalent to a layerwise decidable set $B$. By Prop. 5 the set $A^{*}:=B \cap \mathrm{ML}_{\mu}$ is well-defined and constitutes a canonical representative of the equivalence class of $A$ under $\sim_{\mu}$. If $A$ is already layerwise decidable then $A^{*}=A \cap \mathrm{ML}_{\mu}$. From this, the operator $*$ is idempotent, it commutes with finite unions, finite intersections and complements. 
Proposition 6. If $A$ is a layerwise semi-decidable set then

$-\mu(A)$ is lower semi-computable,

$-\mu(A)$ is computable if and only if $A$ is layerwise decidable.

Measurable maps. We obtain a version of Thm. 3 for measurable maps.

Theorem 4. Assume $Y$ is a complete computable metric space. Let $T:(X, \mu) \rightarrow$ $Y$ be a measurable map. We have:

1. $T$ is $\mu$-recursive $\Longleftrightarrow T$ coincides almost everywhere with an effectively $\mu$-measurable map.

2. $T$ is effectively $\mu$-measurable $\Longleftrightarrow T$ is layerwise computable.

The equivalences are uniform. Observe that while all other implications directly derive from Thm.3, the first one is not so easy as we have to carry out the explicit construction of an effectively $\mu$-measurable function from the equivalence class of $T$. These characterizations show that computability, which trivially implies layerwise computability, implies $\mu$-recursivity and effective $\mu$-measurability.

Let $T$ be $\mu$-recursive: there is a layerwise computable function $T^{\prime}$ which is equivalent to $T$. Let $T^{*}$ be the restriction of $T^{\prime}$ to $\mathrm{ML}_{\mu}$. By Prop. $5 T^{*}$ is uniquely defined.

Integrable functions. We know from Thm. 3 that $A$ is effectively $\mu$-measurable if and only if $A$ is layerwise decidable, which is equivalent to the layerwise computability of $\mathbf{1}_{A}$. As a result, $\mathbf{1}_{A}$ is effectively $\mu$-integrable if and only if $\mathbf{1}_{A}$ is layerwise computable. The picture is not so simple for unbounded integrable functions: although $\int f \mathrm{~d} \mu$ is always computable when $f$ is effectively $\mu$-integrable, it is only lower semi-computable when $f$ is layerwise computable.

Proposition 7. Let $f: X \rightarrow[0,+\infty]$.

- If $f$ is layerwise lower semi-computable then $\int f \mathrm{~d} \mu$ is lower semi-computable (uniformly in a description of $f$ ).

- If $f$ is bounded and layerwise computable then $\int f \mathrm{~d} \mu$ is computable (uniformly in a description of $f$ and a bound on $f$ ).

Hence, we have to add the computability of $\int f \mathrm{~d} \mu$ to get a characterization.

Theorem 5. Let $f: X \rightarrow[0,+\infty]$ be a $\mu$-integrable function. We have:

1. $f$ is $\mu$-recursively integrable $\Longleftrightarrow f$ is equivalent to an effectively $\mu$-integrable function.

2. $f$ is effectively $\mu$-integrable $\Longleftrightarrow f$ is layerwise computable and $\int f \mathrm{~d} \mu$ is computable.

The equivalences are uniform, but a description of $\int f \mathrm{~d} \mu$ as a computable real number must be provided.

We now get a rather surprising result, which is a weak version of Prop. 6 for integrable functions.

Proposition 8. Let $f: X \rightarrow[0,+\infty]$ be a layerwise lower semi-computable function. If $\int f \mathrm{~d} \mu$ is computable then $f$ is layerwise computable. 


\section{References}

1. Edalat, A., Heckmann, R.: A computational model for metric spaces. Theor. Comput. Sci. 193 (1998) 53-73

2. Weihrauch, K.: Computable Analysis. Springer, Berlin (2000)

3. Wu, Y., Weihrauch, K.: A computable version of the daniell-stone theorem on integration and linear functionals. Theor. Comput. Sci. 359(1-3) (2006) 28-42

4. Pour-El, M.B., Richards, J.I.: Computability in Analysis and Physics. Perspectives in Mathematical Logic. Springer, Berlin (1989)

5. Zhang, B.Y., Zhong, N.: $L^{p}$-computability. Math. Logic Q. 45 (1999) 449-456

6. Kunkle, D.: Type-2 computability on spaces of integrable functions. Math. Logic Q. 50(4-5) (2004) 417-430

7. Sanin, N.: Constructive Real Numbers and Constructive Function Spaces. Volume 21 of Translations of Mathematical Monographs. American Mathematical Society, Providence (1968)

8. Edalat, A.: A computable approach to measure and integration theory. In: LICS '07: Proceedings of the 22nd Annual IEEE Symposium on Logic in Computer Science, Washington, DC, USA, IEEE Computer Society (2007) 463-472

9. Bosserhoff, V.: Notions of probabilistic computability on represented spaces. Journal of Universal Computer Science 14(6) (2008) 956-995

10. Martin-Löf, P.: The definition of random sequences. Information and Control $\mathbf{9}(6)$ (1966) 602-619

11. Hertling, P., Weihrauch, K.: Random elements in effective topological spaces with measure. Inf. Comput. 181(1) (2003) 32-56

12. Gács, P.: Uniform test of algorithmic randomness over a general space. Theor. Comput. Sci. 341 (2005) 91-137

13. Hoyrup, M., Rojas, C.: Computability of probability measures and Martin-Löf randomness over metric spaces. Inf. Comput. (2009) In press, available from arxiv.

14. Hoyrup, M., Rojas, C.: Applications of effective probability theory to Martin-Löf randomness. In: ICALP. (2009)

15. Davie, G.: The Borel-Cantelli lemmas, probability laws and Kolmogorov complexity. Annals of Probability 29(4) (2001) 1426-1434

16. Yasugi, M., Mori, T., Tsujii, Y.: Effective properties of sets and functions in metric spaces with computability structure. Theor. Comput. Sci. 219(1-2) (1999) 467-486

17. Brattka, V., Presser, G.: Computability on subsets of metric spaces. Theor. Comput. Sci. 305(1-3) (2003) 43-76

18. Edalat, A.: When scott is weak on the top. Mathematical Structures in Computer Science 7(5) (1997) 401-417

19. Schröder, M.: Admissible representations for probability measures. Math. Log. Q. 53(4-5) (2007) 431-445

20. Zvonkin, A., Levin, L.: The complexity of finite objects and the development of the concepts of information and randomness by means of the theory of algorithms. Russian Mathematics Surveys 256 (1970) 83-124

21. Rojas, C.: Randomness and ergodic theory: an algorithmic point of view. PhD thesis, Ecole Polytechnique (2008)

22. Billingsley, P.: Convergence of Probability Measures. John Wiley, New York (1968)

23. Bressoud, D.M.: A Radical Approach to Lebesgue's Theory of Integration. Mathematical Association of America Textbooks. Cambridge University Press (2008)

24. Müller, N.T.: Computability on random variables. Theor. Comput. Sci. 219(1-2) (1999) 287-299 